\title{
Lymphoplasmacytoid lymphoma elaborating lambda chain paraprotein with amyloid deposition in Sjögren's syndrome
}

\author{
A.W.G. Savage, D.S. Shareef, E.J. Maher, M.H. Bennett and D.J.B. Thomas \\ Departments of Medicine, Radiotherapy and Histopathology, Mount Vernon Hospital, Northwood, Middlesex \\ $H A J 2 R N, U K$.
}

\begin{abstract}
Summary: A woman presented with painful enlargement of her parotid and submandibular glands. She was shown to have the previously unreported combination of idiopathic thrombocytopenic purpura, Sjögren's syndrome, Hashimoto's disease, and myasthenia gravis. Parotid gland biopsy and bone marrow examination showed the presence of a rare lymphoplasmacytoid lymphoma. There was amyloid deposition. in the parotid glands, gums and on muscle biopsy. Immunohistochemical staining of the parotid lymphoma and amyloid was monotypic for lambda light chains, and there was also a lambda chain paraprotein. It is felt that the lymphoplasmacytic lymphoma was responsible for the light chain amyloidosis.
\end{abstract}

\section{Introduction}

Sjögren's syndrome is a multisystem disease that often occurs with connective tissue disorders. ${ }^{1,2}$ It may also be associated with auto-immune thyroid disease and myasthenia gravis. ${ }^{3}$ It is well known that thyroid disease can complicate idiopathic thrombocytopenic purpura (ITP) and more recently myasthenia gravis has been reported in a patient with chronic ITP. ${ }^{4}$ However the case we describe appears to be the first report of a patient who developed ITP, auto-immune thyroid disease, Sjögren's syndrome, and myasthenia gravis. This patient then re-presented with a rare parotid lymphoma associated with light chain amyloidosis.

\section{Case report}

A woman presented in 1967 at the age of 39 with idiopathic thrombocytopenic purpura which was successfully treated by splenectomy. In 1976 Sjögren's syndrome was diagnosed after she developed dry eyes and a dry mouth. During the same year she underwent a partial thyroidectomy for a large goitre, histology of which showed Hashimoto's disease. In 1981 the patient developed diplopia followed by systemic weakness. A positive edrophonium test supported the diagnosis of myasthenia gravis and she responded well to oral prednisolone and pyridostigmine therapy.

In 1986 the patient noticed a painful increase in the size of her parotid and submandibular glands (Figure 1), and complained of profound weakness and

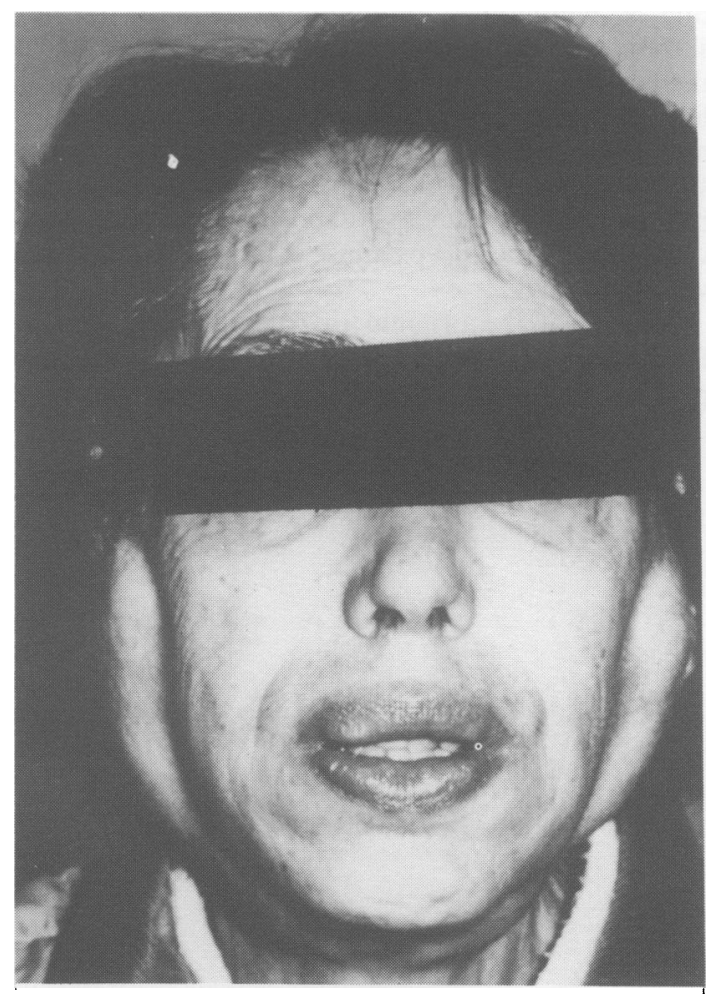

Figure 1 The patient with marked enlargement of the parotid and salivary glands. 
weight loss. Physical examination revealed that she was emaciated with marked enlargement of her salivary glands. There was no hepatomegaly or lymphadenopathy. Rectal and jejunal biopsies were carried out to exclude a gastro-intestinal cause for weight loss. They were considered normal at the time and on review later. In 1987 her weakness persisted and she was investigated further.

\section{Investigations}

Serum IgA level $16.1 \mathrm{~g} / 1$ (normal range $1-1.3 \mathrm{~g} / \mathrm{l}$ ). There was no evidence of immunoparesis. Lambda paroprotein was present in serum and urine. Chest $\mathrm{X}$-ray was normal. ANF positive titre $1 / 80$, DNA double strand antibody negative. Renal and hepatic function were normal.

Parotid gland biopsy showed dense deposition of amyloid (Figure 2). High power magnification of the salivary gland tissue showed that it was markedly atrophic and consisted mainly of ductules and a fairly dense lymphoplasmacytic infiltrate (Figure 3), suggesting a lymphoma. Immunohistochemical staining showed that the cytoplasm of these cells and much of the amyloid tissue stained monotypically for lambda light chain (Figure 4), confirming the presence of a malignant lymphoma. Bone marrow examination showed early involvement by lymphoma. A gingival biopsy revealed amyloid deposition in submucosal vessels and quadriceps muscle biopsy also showed vessel amyloid.

Review of the original thyroid histology showed Hashimoto's thyroiditis but also revealed excessive plasma cell infiltrate, which stained monotypically for lambda light chain. The sections from the splenecto specimen showed the nonspecific changes off idiopathic thrombocytopenic purpura. The patient was treated with chlorambucil $10 \mathrm{mg}$ daily and local radiotherapy which resulted in a partial improvemen. in her symptoms.

\section{Discussion}

Patients with enlarged parotid glands due to Sjögreaìs syndrome run a high risk of developing a lymphoma, estimated to be 48.3 times higher than the expected incidence. $^{5}$ These lymphomas are usually nonHodgkin's B cell types with lymphocytic lymphomas being the commonest salivary manifestation a Waldenstrom's macroglobulinaemia being the co鱼monest extrasalivary lymphoma. Lymphoplasmacytic lymphomas appear to be a very rare complication:

In Sjögren's syndrome the lymphoma is probabigy preceded by benign lymphoepithelial lesions (BLIE), which consist of lymphocyte infiltrates with progres sive destruction of glandular acini and disruption $\mathrm{gf}$ ductal epithelium which may become metaplastio. Salivary gland BLL may occur in Sjögren's syndrome auto-immune thyroid disease, systemic lupłs erythematosus, and a wide range of other aut 5 immune conditions. The more sinister nature of 8 LLE was discovered when the British Salivary Gtand Tumour Panel reviewed 36 cases and found क्षhe 5 patients had developed salivary lymphomas :ard 2 had extrasalivary gland lymphomas. ${ }^{6}$ Schmidt h $\overline{\mathrm{SS}}$ identified a subgroup of BLL in patients wigh Sjögren's syndrome which has a high incidence Of malignant lymphoma development. These lesions a

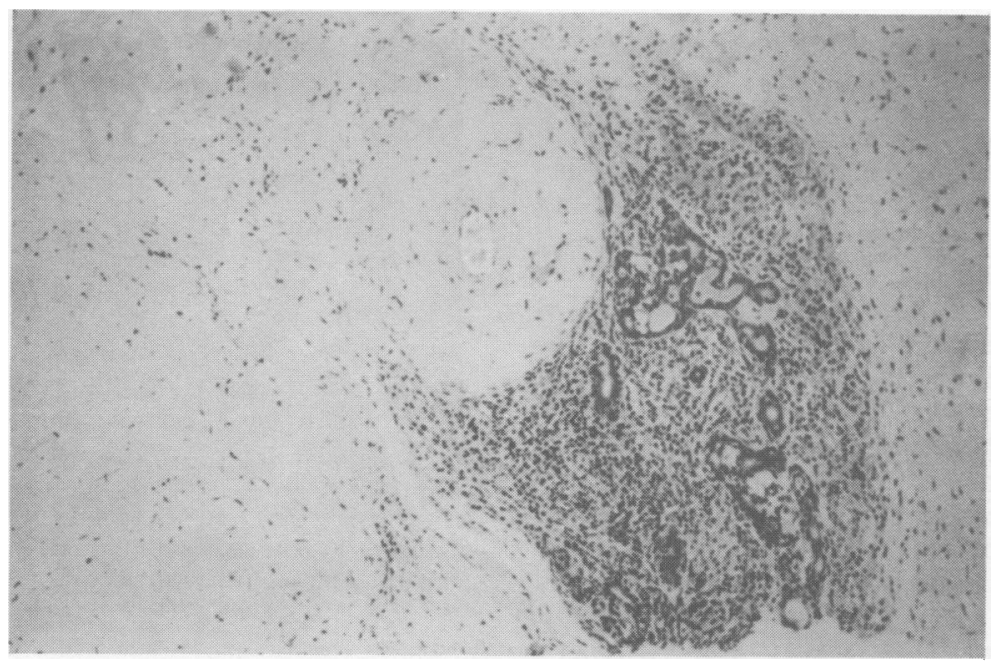

Figure 2 Dense deposits of amyloid in atrophic salivary gland ( $\times 15$, haematoxylin and eosin). 


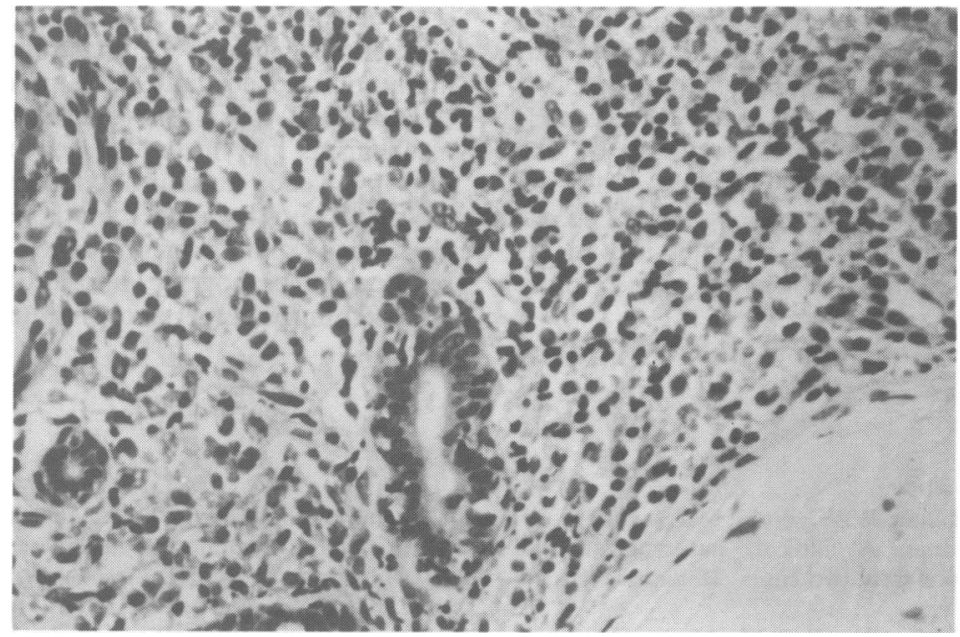

Figure 3 Atrophic salivary gland tissue with ductules and dense lymphoplasmacytic infiltrate $(\times 60$, haematoxylin and eosin).

characterized by extensive confluent lymphoid proliferation and have been termed 'manifest malignant lymphoma'?

It is not clear whether the high incidence of salivary gland lymphoma in Sjögren's syndrome is increased further by the presence of other auto-immune diseases which are known to be associated with salivary BLL. This patient had auto-immune thyroid disease but there was insufficient evidence to diagnose systemic lupus erythematosus. Myasthenia gravis is associated with salivary gland periductal lymphocyte infiltrates but their significance in this condition is unclear. ${ }^{8}$
Parotid gland, muscle and gum biopsy showed amyloid infiltration in this patient which had not been present in rectal and jejunal biopsies two years earlier. The onset of amyloidosis seems to have occurred as the parotid gland enlarged presumably due to the lymphoma infiltration. The lymphoplasmacytoid lymphoma, and much of the amyloid stained immunohistochemically for lambda light chain, and lambda light chain paraprotein was found in the serum. Light chains can be amyloidogenic, a phenomenon most commonly observed with lambda light chains as in this patient. ${ }^{9}$ Review of the patient's thyroid histology with

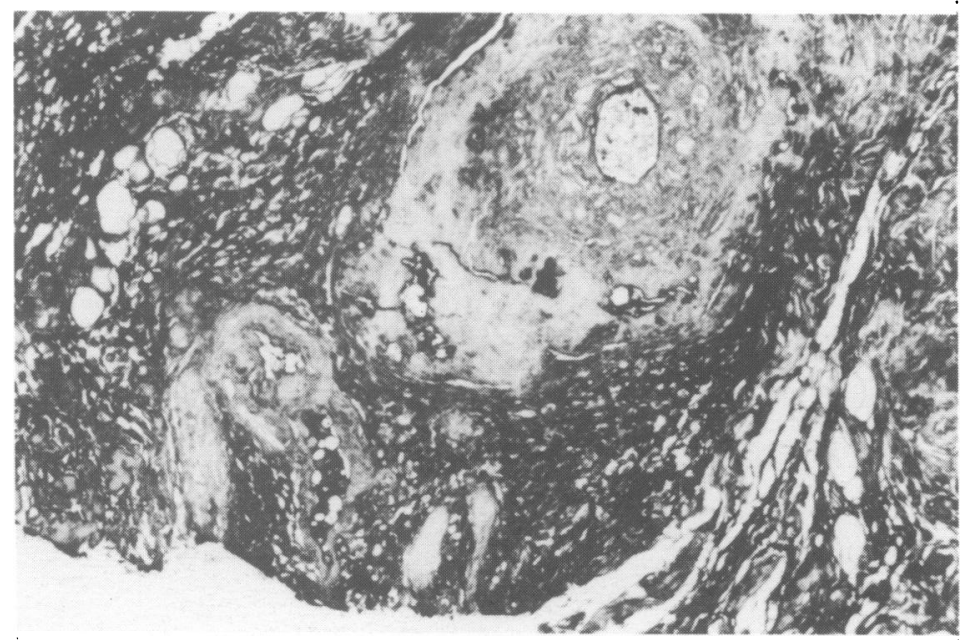

Figure 4 Immunohistochemical staining for light chain showing monoclonal population of lymphoplasmacytic infiltrate $(\times 24$, immunoperoxidase method $)$. 
immunostaining showed an excess of lambda light chain containing plasma cells. This is compatible with studies of serum immunoglobulins in patients with autoimmune disease which have shown that organspecific auto-antibodies are predominantly of one light chain type, either lambda, or kappa in a given patient. Thus plasma cells in patients with Graves' disease and tissue plasma cell infiltrates in patients with Sjögren's syndrome demonstrate lambda chain bias. $^{10,12}$

The evolution of lymphoma is probably slow but

\section{References}

1. Bloch, K.J., Buchanan, W.W., Wohl, M.J. \& Bunim, J.J. Sjögren's syndrome. A clinical, pathological, and serological study of sixty two cases. Medicine 1965,44 : 187-231.

2. Moutsopoulis, H.M., Chused, T.M., Mann, D.L. et al. Sjögren's syndrome. Ann Intern Med 1980, 92: 212-226.

3. Downes, J.M., Greenwood, B.M. \& Wray, S.H. Autoimmune aspects of myasthenia gravis. $Q J$ Med 1966, 137: 85-105.

4. Anderson, M.J., Woods, V.L., Tani, P., Lindstrom, J.M., Schmidt, D. \& McMillan, R. Autoantibodies to platelet glycoprotein IIb/IIIa and to the acetylcholine receptor in a patient with chronic idiopathic thrombocytopenic purpura and myasthenia gravis. Ann Intern Med 1984, 100: 829-831.

5. Kassan, S.S., Thomas, T.L., Moutsopoulis, H.M. et al. Increased risk of lymphoma in the sicca syndrome. Ann Intern Med 1978, 89: 888-892.

6. Gleeson, M.J., Cawson, R.A. \& Bennett, M.H. Benign lymphoepithelial lesion: a less than benign disease. Clin Otolaryngol 1986, 11: 47-51. early stages can be recognized by focal lymphoctite proliferations which demonstrate light chain b immunohistochemically. The process of neoplas change in B cells is not understood, but possible mechanisms may include hyper-responsiveness of-B cells involved in auto-antibody production to growth factors. ${ }^{11}$ An alternative hypothesis is that neoplastic change may result from prolonged local anti-idiotyfic response to pathogenic auto-antibody producing $B$ cells within the target auto-immune organ. ${ }^{12}$

7. Schmidt, U., Hebron, D. \& Lennert, K. Developmentwf malignant lymphoma in myoepithelial sialaden is (Sjögren's syndrome). Virchows Arch 1982, 395: 11-

8. Lindahl, G., Karilefvert, A. \& Hedfors, E. Periduocal lymphocytic infiltrates in salivary glands in myasthenita gravis patients lacking Sjörgren's syndrome. Clin Eocp Immunol 1986, 66: 95-102.

9. Cohen, A.S. \& Connors, L.H. The pathology and biochemistry of amyloidosis. J Pathol 1987, 151: 1- \$0.

10. Jasani, B., Smith, C. \& Williams, E.D. Lambda chain restriction in the diffuse thyroid lymphoid infiltrate in untreated Graves' disease. J Endocrinol Invest 19869 (suppl 3): 101.

11. Theofilopoulos, A.N. \& Dixon, F.J. Murine modबs $\overrightarrow{\&}$ systemic lupus erythematosis. Adv Immunol 198 $\$ 9$ : 269-390.

12. Jasani, B. Immunohistologically definable light restriction in autoimmune disease. J Pathol 1988, 13: $1-5$. 